\title{
Les élus locaux peuvent-ils souder les fragments localisés de l’État?
}

\author{
Gérard Divay \\ École nationale d'administration publique
}

$\mathcal{L}$ e Québec vit actuellement une nouvelle ronde de discussions sur l'opportunité d'accentuer la décentralisation, ronde qui, à ce stade-ci, avec les mécanismes de consultation mis en place, ne soulève pas de tensions publiques marquées. Accouchera-t-elle de résultats plus significatifs qu'au cours des rondes antérieures ? Les structures municipales que les fusions ont voulu renforcer seront-elles le réceptacle de nouvelles responsabilités ? L'issue ne peut être prédite. Mais à première vue, les facteurs de blocage qui ont été souvent avancés pour expliquer les difficultés antérieures de ce genre d'opération semblent toujours

à l'œuvre et sont d'autant plus pesants qu'ils jouent sur plusieurs plans : la marge de manœuvre financière limitée du gouvernement et le refus des responsables locaux d'assumer de nouvelles responsabilités sans compensation financière adéquate, la réticence bureaucratique centrale à faire confiance au palier local, l'inquiétude du gouvernement provincial de se voir aminci alors que le fédéral a plus de moyens pour agir et que le palier local serait renforcé encore plus que par les fusions, l'expertise limitée de certaines institutions locales compte tenu de leur taille et de leur richesse, le confort politique de la répartition entre les deux paliers des arbitrages à faire sur des questions locales (entre autres environnementales)...

En plus de ces facteurs qui tiennent au fonctionnement du système aux plans institutionnel et politicoadministratif, on pourrait avancer une source additionnelle de difficulté qui tient à la conception même de la décentralisation. La façon fort conventionnelle dont elle est présentée ne tient sans doute pas suffisamment compte de manière explicite des pratiques réelles de relation entre le central ${ }^{1}$ et le palier local. Dans le document où le $\mathrm{MAMR}^{2}$ a exposé la démarche de réflexion, en mars 2005, la définition donnée est tout à fait classique : " La décentralisation est le processus qui consiste à transférer des fonctions, des pouvoirs et des responsabilités du gouvernement vers une instance autonome et distincte. Cette instance décentralisée dispose de sources de revenus autonomes et des dirigeants sont élus ». Les maîtres mots, décentralisation, autonomie, transfert, ont pour schème de référence un système à deux paliers relativement indépendants l'un de l'autre, où chacun a sa propre sphère de responsabilité, sans reddition de compte mutuelle. En schématisant, il s'agit en quelque sorte d'un modèle industriel où ce qui est produit par l'un ne l'est pas par l'autre.

La décentralisation est présentée comme un processus de transfert. Le central se départit de responsabilités sur certaines questions et suppose implicitement qu'il ne s'en occupera plus. Lorsqu'il existe des divergences sur le montant de la compensation financière, certains parlent de délestage (on jette pour s’alléger). Certaines perceptions assimilent le central à l'État; le palier municipal est alors considéré comme étant en marge, ou du moins en périphérie, de l’État. La décentralisation participerait alors de la tendance à l'évidement de l'État.

Si l'on quitte le niveau des perceptions et du discours formel sur le partage des compétences pour examiner les pratiques de relations entre le central et le local, on peut se demander - et c'est l'objet précis de cet article - si la décentralisation, tout en garantissant l'exercice 
d'une autonomie formelle, ne vient pas assujettir plus étroitement le local au central en accordant aux élus locaux, plus spécifiquement municipaux, un plus grand rôle dans la coordination de certaines fonctions étatiques. Les municipalités sont des composantes décentralisées de l'État. La décentralisation est aussi un processus de conscription plus ou moins volontaire du local dans les orientations centrales. Le paradoxe n'est surprenant que si l'on garde une vision isolationniste du local. $\mathrm{Si}$, par contre, on prend acte du fait que le local est aussi traversé et mû de forces nationales et internationales sur tous les plans et si l'on retient la thèse maintenant commune que le local dans sa spécificité et sa diversité a un rôle clé à jouer dans le développement, alors la stratégie décentralisatrice se comprend mieux dans son double mouvement d'autonomisation formelle et de conscription volontaire. Elle est perçue et présentée habituellement comme un mouvement centrifuge; elle est aussi dans les faits une stratégie centripète. Le local est devenu trop important pour être laissé aux seules mains des locaux. Pour mettre en œuvre ses orientations dans la diversité dynamique des situations locales, le central a besoin de relais locaux, et ces relais ne peuvent pas être des agents d'exécution. Au contraire, ils doivent disposer d'autonomie et d'une forte légitimité pour mobiliser leur milieu. Leur autonomie se définit essentiellement comme une capacité d'être partenaires du central. La décentralisation devient un passage obligé pour mettre en place une " gouvernance multiniveaux ", conçue comme un mode de fonctionnement de l'ensemble étatique beaucoup plus approprié qu'un modèle hiérarchique pour la gouverne d'une société diversifiée et qui valorise surtout l'innovation comme voie du progrès.

Examinons ce qui nous semble être quelques indices de ce double mouvement, avant d'évoquer quelquesunes des conséquences possibles sur le fonctionnement des institutions locales et centrales.

\section{Le rôle des élus locaux dans l'ensemble des activités de l'État}

Les responsables locaux, toutes catégories confondues, ont déjà une place importante dans les activités du central. Quelques données sur la part des transferts dans le budget du gouvernement du Québec en témoignent. Dans le budget des dépenses 2005-2006, les transferts constituent de loin la supercatégorie pré- dominante (tableau 1). Quatre-vingt-cinq pour cent des 48 milliards de dépenses de programmes y sont affectés. De manière plus spécifique, près de 28 milliards sont transférés à des institutions locales : établissements de santé et de services sociaux, institutions d'enseignement, municipalités. Par ailleurs, 2,4 milliards sont acheminés aux organismes à but non lucratif, dont un bon nombre ont un ancrage local et contribuent de manière explicite, par leurs activités reconnues, à la mise en œuvre des orientations de l'État. Ils constituent en quelque sorte la limite floue des institutions locales (le cas des CPE est un bon exemple à cet égard). Bref, 30 milliards, soit $62 \%$ des dépenses de programmes du gouvernement sont dépensés avec un apport décisionnel local. Bien évidemment, il faut ajouter les quelque 10 milliards de dépenses que les municipalités financent elles-mêmes et les montants moins considérables de revenus autonomes pour les réseaux de l'enseignement, de la santé et des services sociaux. Ces chiffres expliquent sans doute un peu certaines réticences centrales à s'amincir (du moins en \$).

\section{Tableau 1 - Importance des transferts dans les dépenses gouvernementales}

\begin{tabular}{lc} 
Transfert à & (en millions \$) \\
\hline Entreprises & 1004 \\
Entreprises et organismes d’État & 931 \\
Établissement de santé et de services & 14450 \\
sociaux & \\
Institutions d'enseignement & 11744 \\
Municipalités & 1737 \\
Organismes à but non lucratif & 2406 \\
Personnes & 8927 \\
Total Transfert & 41202 \\
Total dépenses de programmes & 48407
\end{tabular}

Source : Québec. Budget des dépenses 2005-2006 vol. 2. Crédit des ministères et organismes (compilation de la supercatégorie transfert de tous les ministères).

Avec de tels chiffres, on ne peut pas dire que les élus locaux ont un rôle marginal dans l'ensemble du fonctionnement de l'État. Cependant, deux séries de réserves s'imposent d'emblée pour donner une juste perspective à ce constat. 
La première concerne la réelle marge de manœuvre des responsables locaux, compte tenu des contraintes exercées par le central et des obligations qu'il impose. Les objets de dépenses et la manière de dépenser, localement, sont balisés par le central et contrôlés plus ou moins fortement selon qu'il s'agisse du réseau de la santé et des services sociaux, des établissements d'enseignement ou des municipalités. De manière encore plus significative pour notre propos, les institutions locales, y compris les municipalités, sont requises de participer à la mise en œuvre de certaines orientations centrales. Sans parler des obligations de fournir certains services, comme dans le domaine de la sécurité; mentionnons par exemple l'établissement obligatoire d'indicateurs de gestion pour contribuer à l'amélioration de l'efficience du secteur public, ou la soumission de plans locaux pour atteindre les objectifs d'égalité en emploi, ou l'envoi d'informations sur les permis émis pour faciliter la lutte contre le travail au noir. Le rôle confié aux instances décentralisées n'en est pas seulement un d'adaptation des services et de réponses à des besoins locaux; il comporte aussi une part de mandats centraux à remplir. Ce constat n’est certes pas unique au Québec; la tendance paraît très généralisée dans les pays de l’OCDE (2003) : « Si on examine les recettes et les dépenses publiques, on observe des tendances globalement divergentes dans l'évolution des dépenses et des recettes des collectivités territoriales. La part de celles-ci dans les dépenses publiques a augmenté dans la majorité des pays. Cependant, les autorités centrales ont de plus en plus essayé de contrer cette tendance en imposant des normes et des niveaux de qualité minimum pour les biens publics fournis à l'échelon local $»^{3}$. Le mot « contrer » laisse entendre une attitude à la fois résignée et machiavélique : on ne peut pas faire autrement que de donner d'une main et de reprendre de l'autre. Dans notre interprétation, il s'agirait plutôt d'un même processus logique : pour maîtriser les évolutions locales et les faire converger vers des objectifs centraux, il faut donner une marge de manœuvre aux responsables locaux.

Quelle que soit l'ampleur des contraintes du central sur les institutions locales (et au Québec elles semblent même moins fortes qu'en d'autres endroits sur certains points, par exemple les indicateurs de gestion), le fait qu'une telle part des dépenses publiques passe sous les yeux d'élus locaux n'est sans doute pas sans incidence sur la capacité de contrôle démocratique des institutions et des activités gouvernemen- tales. La proximité des élus locaux par rapport aux bénéficiaires et par rapport aux gestionnaires en charge des deniers publics est susceptible d'amener un regard plus critique sur les dépenses et les activités, même si le degré et la nature de l'emprise des élus sur les organisations administratives peuvent être très variables. On peut illustrer ce potentiel de regard critique avec un indicateur simple, le rapport entre le nombre d'élus et d'employés (tableau 2); bien évidemment, un indicateur si brut ne dit rien sur l'importance des rôles respectifs, mais il est symptomatique de la proximité politique locale.

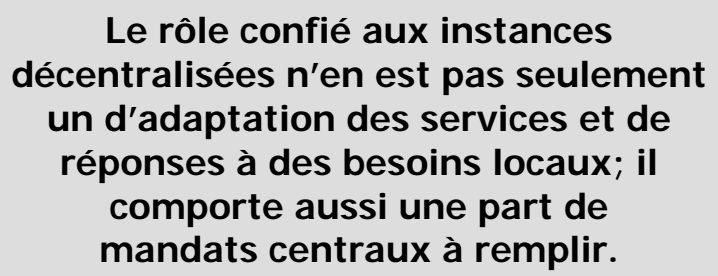

Bien entendu, au-delà des données agrégées, une comparaison devrait se faire entre unités de taille comparable. Mais de ce tableau sommaire, deux constats ressortent : quel que soit le secteur décentralisé, la présence des élus est plus visible dans les institutions décentralisées que dans l'ensemble du secteur public relevant du central. Ensuite, les différences entre les secteurs décentralisés sont importantes, ce qui introduit la deuxième série de réserves sur le rôle des élus locaux.

Le poids des élus locaux varie d'un secteur à un autre. Ces différences tiennent d'abord à des différences institutionnelles : les responsabilités allouées et surtout la capacité de les assumer avec des revenus autonomes diminuent lorsqu'on passe du secteur municipal à celui du scolaire et à celui de la santé et des services sociaux; en outre, dans ce dernier secteur, les élus par la population ne constituent même pas la majorité des membres des conseils d'administration. Par ailleurs, même si ces trois catégories d'élus locaux ont fondamentalement la même légitimité démocratique, elle n'est pas reconnue avec la même intensité compte tenu des différences notoires dans les taux de participation. À titre de rappel, à Montréal, le taux de participation a été de $1,1 \%$ pour les établissements de santé et de services sociaux en 2002 , de $4,5 \%$ pour les élections scolaires en 2003 (à la CSDM), et de $49 \%$ pour les élections municipales de 2001. Compte tenu de ces éléments, il n'est pas surprenant que les 
élus municipaux soient les principaux interpellés dans les discussions en cours sur la décentralisation, même si, en théorie, la décentralisation est supposée être une orientation globale de l'État, donc en principe couvrant tous les secteurs. Mais au fait qu'attend-on des élus municipaux?

Tableau 2 - Ratio élus / employés. Central et secteurs décentralisés au Québec

\begin{tabular}{lccc} 
Secteurs & Nombre d'employés & Nombre d'élus & Ratio élus / employés \\
\hline Public Québec & 69505 & 125 & 556 \\
Santé et services sociaux & 189180 & 738 & 256 \\
Commissions scolaires & 115445 & 1311 & 88 \\
Municipal & & & \\
$\quad$ Municipalités & 74498 & 7882 & 9,4 \\
MRC & 1550 & 1066 & 1,4 \\
Communautés métropolitaines & 42 & 50 & 0,8 \\
Régies intermunicipales & 1316 & 243 & 5,4 \\
OPT & 10865 & 84 & 129
\end{tabular}

Sources. Pour le domaine municipal : MAMR, Prévisions budgétaires des organismes municipaux. Exercice financier 2004. Pour les employés des secteurs public et parapublic : Secrétariat du Conseil du Trésor, L'effectif de la fonction publique du Québec et Effectif du secteur parapublic, données pour 2002-2003, en ETC. Le nombre d'élus pour le réseau de la Santé et des services sociaux est celui qui est prévu, une fois la réforme en cours complétée, selon une donnée fournie par le MSSS.

\section{Les élus municipaux comme coordonnateurs terrain des activités étatiques}

Les attentes officielles sont formulées d'une façon qui laisse sous-entendre des changements potentiels majeurs dans les fonctions des élus municipaux. Les énoncés du document d'orientation Devenir maître de son développement. La force des régions ${ }^{4}$ indiquent que les élus locaux sont appelés à jouer un rôle au niveau local et régional et qu'en même temps, ils doivent aider le central dans l'accomplissement de certaines de ses missions. Deux pistes d'élargissement de mandat se profilent sur chacun de ces deux plans.

Au plan local et régional, le gouvernement reconnaît que « les élus municipaux peuvent le mieux organiser les services »; cette admission justifie une prise en charge éventuelle de nouveaux services. Au-delà de ce rôle conventionnel de prestataire de service, «le gouvernement veut inciter et habiliter les élus municipaux à assumer de plus grandes responsabilités à l'égard du développement économique, social et culturel des régions ». Comme toutes les dimensions du développement sont visées, rien des choses locales et régionales n'échapperait aux préoccupations des élus municipaux. Leur participation à certains aspects du développement est enracinée depuis longtemps; mais le fait qu'on veuille les mettre à contribution sur tous les aspects signale un changement de cap. Certes, à ce stade-ci, le dosage des responsabilités entre les services aux citoyens et la prise en charge du développement n'est pas explicité. Dans les faits, hors la discussion sur la décentralisation, il est curieux de constater que le gouvernement tient à garder l'organisation d'une part importante des services publics (santé et services sociaux) dont la dimension locale est significative, alors qu'il confie aux élus municipaux des régions le développement, que plusieurs politiques centrales influencent fortement, même si la mobilisation des facteurs endogènes de développement suppose une solide expertise collective locale.

Par rapport au central, on attend des élus locaux qu'ils jouent un rôle de coordonnateurs et d'agents de changement de l'État. La coordination peut s'exercer dans les services et la mise en œuvre des politiques. Dans les services, les élus locaux, via un mandataire 
OBNL, le CLD, peuvent mettre en place un guichet unique de services aux entreprises. Dans les politiques, le document mentionne la coordination des actions en faveur de l'immigration. Parler d'un rôle d'agent de changement de l'État pour les élus municipaux peut apparaître comme une interprétation un peu audacieuse des orientations officielles. Mais les mandats proposés montrent que le central s'attend à ce que les élus municipaux l'aident à se réformer sur des aspects significatifs de son fonctionnement. Les CRÉ (Conférences régionales des élus) devront « évaluer les organismes de planification et de développement aux paliers local et régional dont le financement provient en tout ou en partie du gouvernement ». Ils ont aussi à contribuer à l'adaptation des normes et des programmes gouvernementaux aux réalités régionales. Les élus locaux deviendraient ainsi instrumentaux dans le passage du mur à mur au sur mesure, alors que le fonctionnement du système politico-administratif des relations locales-centrales montrent qu'ils peuvent autant avoir des positions universalistes que particularistes selon les dossiers.

Avec ces mandats, les élus municipaux participeront plus explicitement à la vie de l'État. Pour être des coordonnateurs et surtout des agents efficaces de changement, ils ont besoin d'un pouvoir autonome; sinon, ils auraient autant et pas plus d'influence que des consultants. L'État central a besoin de les avoir comme partenaires capables de fournir un apport original ou, pour reprendre une expression courante, d'avoir « une valeur ajoutée » politique. D’ailleurs, le thème du partenariat est omniprésent. Les CRÉ peuvent s'engager dans au moins trois types de partenariat. Le document sur la nouvelle gouvernance régionale souligne que près de 250 ententes ont été conclues depuis 1994 avec les régions, sans parler du contrat de ville avec Montréal. Le partenariat est aussi un leitmotiv du plan stratégique 2005-2008 du ministère des Affaires municipales et des Régions ${ }^{5}$.

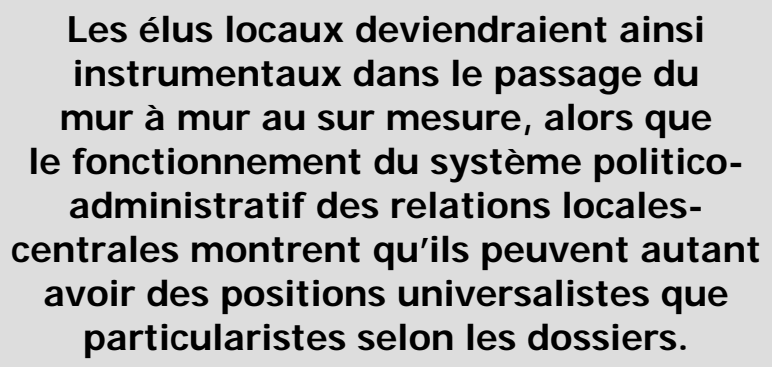

\section{Repenser la décentralisation}

L'interprétation des tendances en cours qui vient d'être brièvement esquissée et qui tient compte autant des discours que des pratiques dans les relations entre le centre et le local amène à reconsidérer la décentralisation dans ses objets, ses notions et ses conditions de mise en œuvre, ainsi qu'à s'interroger sur le fonctionnement politique actuel de l’État.

La décentralisation n'est pas dans la pratique un simple transfert qui retranche au centre et ajoute au local. Elle porte plutôt surtout sur la gouvernance multiniveaux, sur les relations entre le centre et le local. Elle veut renforcer l'autonomie locale, dans la mesure où cet ajout d'autonomie permet aux élus municipaux de devenir des partenaires dans la mise en œuvre d'orientations gouvernementales (le cas échéant dûment transigées). Il ne s'agit donc pas de l'autonomie au sens de liberté de faire ou ne pas faire, à la guise locale. Cette autonomie se définit davantage comme une obligation d'initiative.

L'État central a besoin de ces initiatives locales; la singularité des situations locales suppose une diversification des moyens pour atteindre des fins semblables. Dans les services, l'autonomie locale permet éventuellement plus d'efficience; pour le développement, elle devient une condition d'efficacité de l'action gouvernementale. La valeur ajoutée de l'initiative des élus locaux déborde "l'adaptation aux besoins locaux »; elle vient d'une capacité de combiner diverses mesures gouvernementales et de les arrimer à la mobilisation des forces du milieu, d'anticiper et de rendre plus synergiques les effets de diverses mesures sectorielles. Dans une approche partenariale entre niveaux de gouvernement, elle n'est pas octroyée sans condition, elle s'obtient et se démontre par un apport spécifique. Dans cette perspective, le rapport devient étroit entre la responsabilité et l'imputabilité; comme l'énonce si clairement Lemieux ${ }^{6}$ : «L'imputabilité consiste à rendre des comptes au centre, alors que la responsabilité consiste à répondre aux attentes de la base ». Dans la décentralisation partenariale, les élus locaux doivent aussi répondre aux attentes d'en haut, plus exactement, ils doivent arrimer les orientations gouvernementales et les préférences locales; ils sont des partenaires médiateurs et mobilisateurs.

Donner un tel rôle aux élus municipaux n'est pas sans conséquences sur le fonctionnement politique de 
l’État. Évoquons-en sommairement quelques-unes en conclusion, même si une discussion plus longue serait de mise, surtout que certaines tendances observables ne sont pas uniques au Québec.

\section{L'élection directe des préfets des MRC va sans doute atténuer la perte de pouvoir et les difficultés d'arbitrage local/ régional propres à une instance de second degré.}

En premier lieu se pose une question de légitimité des élus municipaux dans leurs mandats centraux. Il est à noter que, jusqu'à maintenant, ces mandats toucheront surtout les CRÉ, composées principalement d'élus municipaux et non d'élus directs au niveau régional. L'élection directe des préfets des MRC va sans doute atténuer la perte de pouvoir et les difficultés d'arbitrage local/régional propres à une instance de second degré. Ensuite, quelle place reste-t-il pour les élus centraux, les députés ? Ils sont membres de la CRÉ, sans droit de vote. Certes, comme membres de l'Assemblée nationale, ils verront le rapport de reddition de comptes que soumettra la CRÉ. Mais dans leur travail de circonscription, se verront-ils davantage cantonnés au rôle d'intercesseur entre les citoyens et les services gouvernementaux, alors que les enjeux de développement et les contributions étatiques seront davantage traités par les élus municipaux conjointement avec l'exécutif central ? Ensuite, dans quelle mesure un plus grand rôle pour les élus municipaux va-t-il modifier la dynamique des relations avec la société civile, entre l'État central et les organismes sans but lucratif qui travaillent à sa marge, souvent pour son compte, entre l'État central et les représentants associatifs des divers secteurs et groupes d'intérêt? Dans certaines circonstances, les groupes locaux, avec l'appui du central, ont pu être des leviers de changement dans les actions publiques locales; cette multiplicité des sources d'innovation sociale locale va-t-elle être maintenue, diminuée ou renforcée ? Dans les CRÉ, les élus municipaux doivent faire une place aux représentants des divers secteurs socio-économiques.

Les conséquences du nouveau dispositif institutionnel qui intègre davantage les élus dans la mouvance étatique vont sans doute beaucoup dépendre de la façon dont les élus vont assumer leurs rôles. S'ils s'en tiennent à un rôle de décideurs administrateurs d'organismes services ou s'ils utilisent à fond leurs habiletés de médiateurs, le jeu sera plus ou moins ouvert et les attentes officielles à leur égard plus ou moins comblées.

\section{Notes et références}

1 Dans ce texte, le central réfère au gouvernement du Québec, palier qui a juridiction sur les municipalités. Une réflexion plus globale sur l'État au niveau local devrait inclure le fédéral.

2 Ministère des Affaires municipales et des Régions (2005). Projet gouvernemental d'autonomie régionale et municipale. La décentralisation, $10 \mathrm{p}$.

3 Revue économique de l'OCDE, n 36, 2003/I, «Les relations financières entre l'État et les collectivités locales », I. Joumard et P. M. Kongsrud.

4 Gouvernement du Québec (2004). Devenir maître de son développement. La force des régions. Phase 1 une nouvelle gouvernance régionale, $37 \mathrm{p}$.

5 Ministère des Affaires municipales et des Régions (2005). Plan stratégique 2005-2008, 31 p.

6 Lemieux, V. (2003). « Décentralisation et responsabilisation », Organisations et territoires, vol. 12, n 3 , automne, p. 11-16. 